\title{
Muerte materna por embolia de liquido amniótico
}

\section{Por JORGE R. FIRPO}

En el año 1941, Steiner y Lushbaugh señalaron, en la autopsia de una parturienta muerta durante el parto, la existencia de embolías múltiples constituídas por los componentes del líquido amniótico, con lo cual incorporaron una nueva entidad nosológica a la patología de la gravidez.

\section{Frecuencia}

A la comunicación inicial de ocho casos de sus descubridores, siguió otra de dos nuevos de los mismos autores. Desde entonces la literatura se ha ido enriqueciendo con un caso de Hemmings, uno de Goodof, uno de Burt, tres de Gross y Benz, uno de Wyalt y. Goldenberg, uno de Jennings y Stofer, dos de Barron, Sturley y Lindsay, uno de Watkins, uno de Seltzer y Schman, uno de Shotton y Taylor, uno de Steiner, Lushbaugh y Frank, uno de Crowder y Bills, uno de Woiner y Reid, uno de Marcuse y Duson, uno do Newberger y últimamente tres de Mallory y colaboradores. Sin embargo. the estos relatos, que alcanzan al númrro de 31. no pueden extraerse con clusicnes sobre la mayor o menor fic cunncia, porque adomás de tratars. de casos aislados, es una afección cuyo diagnóstico sólo puede hacerse mediante la autopsia y aún esta misma debe ser hecha con detenimiento para poder descubrir las lesiones histológicas pulmonares; agrégase la posibilidad de que algunos casos, pasando desapercibidos en su verdadera naturaleza, sean rotulados de otra manera y por último queda todavía la alternativa de casos no fatales cuyo diagnóstico puede permanecer ignorado.

Por consiguiente, es dificil precisar la frecuencia. En la clasificación que sobre choque obstétrico hace Feeney, es probable que el segundo grupo corresponda a este tipo. El choque es, según Johnsor, la causa más frecuente de muerte súbita en obstetricia y debe tenerse present la similitud clínica de ambos procesos. Sheehan, estudiando 147 muertes, Scoll 82 y May y Winter 81, no comprobaron este accidente, pero cabe preguntarse si el estudio histológico de los pulmones fue hecho en forma correcta, ya que la revisión de los cortes histológicos de algunos casos oscurcs podría senalar, según Steiner, características embólicas. Los descubtidores dieron como frecuencia uno por 8.000 partos. Llama la atención que Gross y Benz hayan encontra- 
co tres casos en un año, sobre 1.200 partos, que por otra parte, fueron las tres únicas muertes maternas ocurridas en ese lapso, lo que eleva la trecuencia a uno por cada 400 partos.

Todo lo expresado confirma que cunlquier cifra concreta ha de ser in. sutura; si bien no debe ser sobreva. Intadu, tampoco se la ha de considetrit una rareza y deberá ser tenida en cuenta en todas las muertes maternas de causa poco clara y especialmente cuando se las rotule como choque obstétrico.

\section{Etiopatogenia}

El proceso se instala a raíz del pa. scie de líquido amniótico dentro de la circulación. Experimentalmente se ha producido un cuadro similar, inyectando liquido amnlótico no filtrado por vía endovenosa en conejos y perros. Es evidente que en la parturienta de be establecerse una comunicación entro el líquido amnlótico y los senos sanguineos maternos, capaz de permltir el pasaje. En apoyo de esta hipótesis se ha señalado on algunos casos la presencia de sangre en la cavidad arnniótica y en otros el hallazgo de componentes del líquido amniótico como pelos, hunto sebáceo y escamas, dentro de las venas de la pared del útero y parametriales. Leary y Hertig, examinando placentas han encontrado on algunas de ellas, células escamosas en ubicaciones anormales: entre el amnios $y$ el corion $y$ hasta on un caso en los sinusoides de la decidua, sin embargo esas mujeres no manlfes taron ninguna morbilidad. Pero frente a estos casos en que se ha comproba. do la existencia de una puerta de en. trada, representada por laceraciones, desgarros, heridas, hay otros on los cuales no ha sido posible encontrar soluciones de continuidad, al menos aparentes, en la pared uterina.

Hay una serie de factores que predisponen a ese pasaje: senos matemos muy próximos a la cavidad amniótica, incisiones o desgarros de cuello. zonas necróticas de las envolturas en los casos de feto muerto; la edad avanzada podría desempeñar algún papel. pues salvo el caso de Seltzer y Schuman, en la mayoría se trataba de mujeres añosas. Todas estas situaciones confirmarian un terrenos que ante la concurrencia de otros factores estable cería una situación de verdadera fa. cilidad para la invasión circulatoria por el líquido amniótico. Estos elementos edesencademantes:, capaces de forzar al líquido a través de una efracción, estarían representados por contracciones uterinas violentas, características que señalan casi todas las his. torias clínicas como precediendo a la aparición de los primeros síntomas. La rotura alta de la bolsa, que hace al líquido escuritir comprimido entre las membranas y la pared del útero, lo mismo que pujar con energía exce siva cuando la cabeza, apoyada en el piso pelviano, bloquea la salida del líquido amniótico, desempañan también un papel etiológico.

Landing, estudiando úteros extirpados por diversas razones durante el parto o en la cutopsia, afirma que el líquido amniótico solamente entra en la circulasión materna por el camino de vasos anormalmente abiertos: operación cesárea, rotura del útero, placenta accreta y desprendimiento prematuro normoplacentario. 
El por qué de la muerte de la enferma no está perfectamente aclarado. La embolía es alaramente responsable cuando abarca un amplio territoriso, con lo cual, bloquea mecánicamente la circulación, pero en los casos en que la zona comprometida es pequena, debe buscarse otra explicación: un reflejo vasoconstrictor en el pulmón. corazón y sistema vascular; atras ve ces se agrega un edema agudo de pul. món y también pueden tener su parte las hemorragias uterinas que con mucha frecuencia acompañan al proceso que nos ocupa. Watkins admite la posibilidad de una reacción de tipo anafiláctico. Cualquiera de estos hechos es suficiente por sí mismo para producir la muerte.

En una revista de la literatura, Dumont, se pregunta si en algunos de los casos descritos, la causa de la muerte fue realmente la embolía o si ésta constituyó solamente la repercusión de otro accidente que sería el original; en otras palabras, si la embolia ha sido todo el síndrome o una parte de él; en apcyo de estas ideas señala dos casos de rotura uterina y la existencia de una cesárea en otro. También la atonia que acompaña a esta complicación, puede no ser secundariá, sino primitiva y forvorecer el pasaje de líquido amniótico. Pero sea verdad cualquiera de estas razones o cada una de ellas, sogúin los casos, lo real oo que se produce un pasaje de elemen tos del líquido amniótico a la sangre. que éstos se atascan en el pulmón y que la enferma muere en corto plazo.

\section{Anctomia patológica}

Los hechos más significativos en el examen anatomopatológico son dos:
1) Existencia de numerosos cuerpos extraños en los capilares, arteriolas y arterias pequeñas pulmonares; 2) $\mathrm{Ca}$ racterísticas especiales de la sangre.

El examen macroscópico es poco ilustrativo: al abrir el vientre en uno de los casos de Gross y Benz, se encontraron 200 gramos de sangre extravasada; estos derrames sanquíneos se pueden encontrar en el tejido preperitoneal, en forma de equímosis en la raíz del mesenterio, ligamento ancho, trompas, ovarios y crún en el tojido celular subcutáneo. Los órganos genitales, útero y onexos, se muestram edematosos y a veces con extensas trombosis en algunos vasos, mientras que en otros la sangre permanece líquida, sin coagular.

El bazo señala una hiperemia activa, contrastando con la gran palidez del hígado, debida al edema de las células hepáticas. En el corazón se ha encontrado dilatación derecha y la sangre contenida en él con la característica ausencia de coagulación. En el pulmón puede haber edema y en el árbol bronquial espuma y mocos sanguinolentos.

El examen histológico señala detalles característicos, tánto que sólo me diante él puede certificarse el diag. nóstico.

El pulmón muestra numerosas embolías por partículas materiales de líquido amniótico, ubicadas en los capilares, artertolas y arterlas pequeñas; en el corte se ve que algunos vasos están exangües y otros ocluídos por esos elementos, a los que se agregan polinucleares. Estos acúmulos están constituídos por una mezcla de detritus granulosos acidófllos; fibrillas basófllas, encerrando a veces escaros 
gionuios rojos, escasas eprieliares y detritus de tinte bilioso (meconio); las escamas y el lanugo pueden estar presentes $y$ mezcladas en proporciones variables con vérnix caseoso. Los leucocitos adoptan una disposición que parece ser característica: están alinea. dos en hileras paralelas como un pei. ne: su origen es oscuro y según Steiner, Lushbaugh y Frank, su presencia en los núcleos embólicos puede tener uno de los tres significados siguientes: 1) Movilización extraordinariamente rápida, ya que también ham sido encontrados en un caso en que todo el pro ceso duró apenas 15 minutos; 2) Al pasar el émbolo a la sangre los enredó dentro de él, arrastrándolos hasta esa localización pulmonar; 3) Que la embolia haya precedido largamente al comienzo clínico de los síntomas. En cuanto al endotelio vascular no se observa ninguna anormalidad. La mayoría de los alvéolos están vacios, excepto algunos que contiemen pequeñas cantidades de líquido.

Al estudiar el útero se han podido encontrar grandes masas de escamas eptleliales on el interior de un seno sanguíneo ubicado en las proximida. des del endometrio.

En el examen histológico del hígado se vió un líquido albuminoso que establecía una pequeña separación entre el endotelio sinusoidal y los cordo nes hepáticos, las células hepáticas ediematiosas y los sinusoides estrechados con muchos polinucleares y escasos eritrócitos.

La sangre presenta un detalle característico: no coagula. Centrifugada (Gross y Benz), se separa en tres capas en vez de las dos con que lo hace normalmente: por encima de la segun-

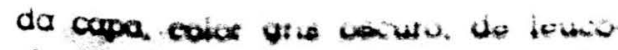
citos, w ve una wons vallowa de color

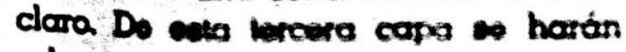

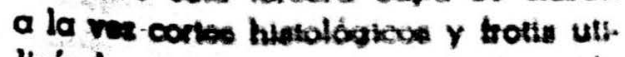
lixtindose thelones con of this do Wright: low cortos wor tiado con a'.

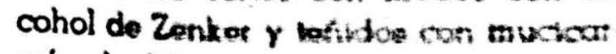
min de Marer liqwe idontitica el mis

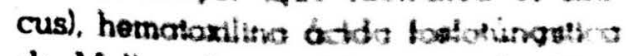
de Mallory para difoterator la labt? na, del mucus $r$ examas epteliates y con hematailina eastio para la propósitos de rutina. Do wa mara. puede vere que eata vercera copa es to constturda por matmial acdidalo fincmente gramulaso, contminado queños rocimas de leucoction in co. denas de 10 a 20 elementos mucut teñido de rosa por el muctommin: ró nulos finos de color amatllo oro fib. lis!) y escamas vetildas do rojo por la eosina. En cuanto a la copa medu; formada por la crema de leucoctios no mostró delalles pariculares ni en los frotis ni en los corter.

Esta sedimentactón de la ecmoro. an tres capas, podila conalderada po tognomónica do la entermedad: como los constituyentes dol liquido cmniól: co son de baja densldad. depositon en una tercera capa por encima da la sogunda de leucoctios

Se ha demostrado que ol liquilo amnlótico poses una marcada octivi. dad coagulante, la cual jugaria un papel en el meconiemo normal do ho.

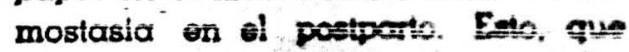
aparentemente extaria on contradic: clón con las manifeatactones hemontó. gicas de la embolia de liquildo amnits. tico y con la talta de coosulación do la sangre extraida por punction del corazón derecho, explica, axún Weiner y Reld, porque al entras of 
terna se produciría una intensa coagulación intravascular, lo que traería como resultado una verdadera afibrinogenia por la utilización de todo el fibrinógeno de la sangre.

Este procedimiento de examen de la sangre tiene la ventaja de solucionar el problema planteado por aquellos casos en que no se puede hacer la autopsia, ya que podrá extraerse la sangre del corazón derecho, mediante aspiración.

En resumen, los elementos anatomopatológicos característicos se encuentran en el pulmón, con las embolias múltiples y la característica disposjción en peine, de los leucocitos y en la sangre, con dos aspectos fundamentales: incoagulabilidad y sedimentación en tres capas.

\section{Sintomatología y diagnóstico diferencial}

El accidente comienza en las postr:merías del parto o en seguida de él. En esto coinciden todos los casos, con excepción del de Crowder y Bills, en que se produjo 10 minutos después de una rolura prematura de las membra. nas, en cusencia de trabajo de parto y en una cesárea que se practicó antes de la fecha de término (Lushbaugh y Steiner), por hipertensión y placenta previa. La iniciación es más o me nos brusca, sin causa aparente o al hacer un esfuerzo (caso de Wyatt y Goldenberg al subir a la camilla) y hace pensar en un choque o colapso. Algunas veces ha sido precedida por un vómito bilioso: en casi todos los casos, un tiempo antes se presentaron contracciones uterinas violentas $y$ nasta tetanitormes. La enferma, cuando nada hacía esperarlo, siente un desvanecimiento, disnea, escalotrios, taquicardia, hipotensión, cianosis, lo que configura un cuadro de choque. Puede haber algunas convulsiones, el estado de desasosiego persiste, la piel se muestra fría y viscosa la hiperpnea va en aumento, hay desviación conjugada de los ojos hasta que se instala el coma. La muerte so presenta en un piazo variable, pero que siempre es corto, oscilante entre cinco minutos (caso de Watkins) y una hora del comienzo de los sintomas. La muerte se produce en coma o colapso y puede ir acompañada de una pérdida sanguínea de intensidad variable. Estas pérdidas de sangre pueden pro sentarse no solamente en los genitales, sino en otros lugares: en el caso de Hemmings, la enferma tuvo hemo rragias nasales y subcutáneas.

La radiografía de pulmón, como método auxiliar de diagnóstico, fue practicada en un caso por Seltzer y Schuman, comprobándose un tranco aumento de densidad.

En cuanto al método de Gross y Renz, de sedimentación de la sangre, en tres capas, tiene el grave inconveniente de que la sangre debe extraerse del corazón derecho o de la carva, lugares de evidente riesgo para la punción en una mujer viva.

Como puede notarse, esta sintomatología se presia a confusiones diagnósticas desde el punto de vista clínico:

Hemorragias postparto. Su frecuente coincidencia, lleva la atención hacia los genitales e inclusive, se ha lle gado a taponar el útero atribuyéndosele al principio la responsabilidad en 
la muerte de la enferma. Ya hemos so nalado que Dumont plantea el into rrogante de si esta hemorragia es realmente secundaria a la embolia o si la precede. De Loe ha sugerido, que si existen formas subletales podrían tener como síntoma principal la ato nía postparto. Para establecer el diagnóstico diferencial se verificará lo más exactamente posible, la cantidad de sangre perdida, para ver si realmente justifica la gravedad del cuadro. Otro detalle podria consistir en comprabar 8i la hemorragia está relativamente alejada del comienzo de los síntomas generales.

Embolia pulmonar trombbalca. Po drá descartarse por la cusencia de en. fermedad embolizante y por su preco cidad de aparición.

Eclampeia ain convulsiones. Puede plantearse este diagnóstico en los casos con síntomas de edema pulmonar $y$ especialmente si se admite que el -asma cardíaca, en el parto, puede ser una manifestactón de toxemia. La ausencia de antecedentes de patolo gía cardiovascular y la falta de signos de gestosis serán los elementos diferenciales a considerar.

Aspiración de sangre o contenido gúsutrico. Se la deberá considerar en casos en los que se haya aplicado anestesia, pero se la descartará por el control cuidadoso durante el sueño quirúrgico o si la anestesia ha evolucionado sin dificultades. Algunos anes tésicos como el protóxido de ćzoe,po drían ser inconvenientes por su acción depresora.

Choque. Es el cuadro más semejante. Sin embargo, si la enterma ha sido observada carrectamente, durante el parto y en ausencia de manjobras vio lentas, se está en condiciones de com. probar si realmente han existido las corusas capaces de desencadenar un choque.

\section{Pronbetico y tratamiento}

Desgraciadamente la situación es irreversible: una vez instalada, term:na con la muerte; todos los recursos terapéuticos han tracasado. En los primeros casos, la similitud con el cuadro de choque obstétrico. hizo que se utilizara la terapéutica carrespondiente a él.

El tratamiento, como lo califica apropiadamente De Loe, es teórico y sólo puede ser sintomático. Para evitar el reflejo cardíaco, la atropina puede ser de utilidad; esta acción beneficiosa se ha comprobado experimentalmente: administrándola a perros, antes de la inyección endovenosa de líquido am. niótico (Shotton y Taylor) disminuye la gravedad de las reacciones; debe darse en grandes dosis y arociada a la morfina. La paparverina combate a su vez el espasmo vascular y las inhalaciones de oxígeno alivian la disnea. En camblo, las transfusiones de sangre o plasma están contraindicadas, porque aumentan el edema pulmonar.

Seltzer $\gamma$ Schuman han presentado un caso no fatal en una mujer joven, 25 años, en la que ocurrió a continua. ción de un fórceps. Hicieron la medicaclón ya cttada y agregaron dicumarol; la enferma luégo de cinco días azarosos de altibajos violentos, se recuperó fincimente. Este caso, Junto con el que ủltimamente ha presentado Miles, serían los dos únicos de toda la serie que no terminaron con la muerte. $P_{\theta}$ ro, en ambos puede hacerso la misma 
objecion: si bien la descripcion chinica corresponde exáctamente a la sintomatología de los demás casos, falta la comprobación histológica; tratándose de un diagnóstico clínico, la única do cumentación está representada por radiografías de pulmón que muestran un intenso aumento de la densidad de la trama pulmonar. Mendelson hace la objeción de que podría haberse tratado de una aspiración de sangre $y$ contenido gástrico, pues ocurrió duran. te una anestesia. Seltzer y Schuman, en su respuesta desmenuzan cuidadosamente el diagnóstico diferencial y llegan a la conclusión de que realmente se trataba de una embolia de líquido amniótico, atribuyendo el éxito, no solamente a la terapéutica, sino también a que la embolía no fue masiva y se produjo en una mujer joven y de muy buena constitución física, juventud y fortaleza que le permitieron soportar la grave complicación.

Es un accidente que no puede ser previsto, pues en varias oportunidades se ha instaiado en casos que evolucionaban normalmente y en ausencia de maniobras intempestivas; el partero no puede por lo tánto, ser responsabilizado. Queda por admitir la existencia de formas frustras que no llegarían a producir la muerte y cuyo diagnóstico quedaría ignorado.

De todas maneras la evolución que da librada al caso por sí mismo sin que tome mucho lugar el tratamiento indicado por el médico. Si algo puede hacerse como profilaxis, es evitar algunas de las que parecen causas predisponentes: contracciones tetánicas, pujar con violencia cuando existe mucho líquido retenido porque la cabeza apoya en el piso pelviano, las roturas altas de la bolsa de las aguas, evitar efracciones de la pared uterina especialmente en el cervix.

Pero todas estas no dejan de ser consideraciones más teóricas que prácticas y en la realidad podemos afirmar que el tocólogo posee muy pocos recursos para hacerle frente.

\section{Fábrica Ortopédica “EL PIE” Doctor LEOPOLDO LOEW}

Doctor: Cuando usted necesite una faja de tipo especial - sobre medidas, pida el formulario respectivo.

Fajas para maternidad, para antes y después del parto.

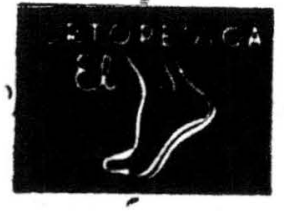

Fajas abdominales. Corsets ortopédicos. Sostén para apósito rectul. Piernas artificiales metálicas. Plantillas para pies planos y juanetes. Bastones. Muletas.

Calle 35, número 7-93. - Teléfono No. 57-845 - Bogotá 